Durch das PBG materialisiert der Gesetzgeber den konstitutiven wehrverfassungsrechtlichen Parlamentsvorbehalt. Es schafft dadurch Rechtssicherheit und Rechtsklarheit für alle an der konstitutiven Entscheidung des Bundestages Beteiligten. Rechtliche Grauzonen werden wenn schon nicht völlig beseitigt, so doch aufgehellt. Streitfragen werden - wie das umstrittene Rückholrecht - geklärt. Das ist nicht das Geringste, was das schmale Gesetz leisten kann.

Das PBG wird - so darf vermutet werden - nicht das letzte Wort des Bundestages zur gesetzlichen Ausgestaltung seiner Beteiligungsrechte sein. Die fortschreitende Integration deutscher Streitkräfte und die in diesem Zusammenhang zu erwartende Praxis in NATO, WEU und EU werden in nicht sehr ferner Zukunft erneut die Frage aufwerfen, ob die gesetzliche Ordnung des konstitutiven wehrverfassungsrechtlichen Parlamentsvorbehalts der Wehr- und Bündnisfähigkeit der Bundesrepublik Deutschland, aber auch den Rechten des Parlaments angemessen Rechnung trägt.

Vor dem Hintergrund der fortschreitenden und sich vertiefenden Integration militärischer Strukturen wird der Gesetzgeber in Zukunft immer wieder die Frage beantworten müssen, ob die militärische Einsatzrationalität im Bündnis stärker zu gewichten ist als das vorherige konstitutive Beteiligungsrecht des Parlaments. ${ }^{105}$ Eine vorschnelle Beschränkung der Beteiligungsrechte des Bundestags aus Gründen der Rücksichtnahme auf Bündnisstrukturen ist dabei nicht zu befürworten. Der konstitutive Parlamentsvorbehalt in seiner konkreten Wahrnehmung durch den Bundestag ist der Bundesrepublik Deutschland bislang gut bekommen. Zwingende Gründe für eine „regierungsfreundliche“ Reform des PBG sind gegenwärtig nicht ersichtlich.

105 Vgl. auch Roman Schmidt-Radefeldt, a.a.O. (Fn. 63), S. 201 (S. 206); Andreas L. Paulus, a.a.O. (Fn. 1), S. 307; Markus Rau, in: AVR 44 (2006), S. 93 (S. 112 f.); MdB Hans-Ulich Klose (SPD), a.a.O. (Fn. 72), S. 22 ff.

\title{
Der Entscheidungsprozess über den Bundeswehreinsatz zum Schutz der Wahlen im Kongo*
}

\author{
Jan Ryjáček
}

Seit dem Urteil des Bundesverfassungsgerichts vom 12. Juli $1994^{1}$ stehen die Auslandseinsätze der Bundeswehr unter einem konstitutiven Parlamentsvorbehalt und werden von einer breiten gesellschaftlichen und politischen Diskussion begleitet, so zuletzt die Einsätze in Afghanistan, im Libanon und im Kongo. ${ }^{2}$ Für das Entscheidungsverfahren, das sich im

\footnotetext{
* Dieser Beitrag ist Teil des Dissertationsvorhabens zur Rolle des Deutschen Bundestages in der Europäischen Sicherheits- und Verteidigungspolitik, gefördert durch die Grantagentur der KarlsUniversität zu Prag (GAUK), Projektnr. 257196.

1 BVerfGE 90, S. 286.

2 Zur Diskussion vgl. auch den Beitrag von Dieter Wiefelspütz in diesem Heft der ZParl.
} 
Laufe der 1990er Jahre entwickelt hat, stellen die Entstehung und der Aufbau multilateraler Eingreifverbände im Rahmen der NATO (NATO Response Force) beziehungsweise der EU (European Battle Groups) in den letzten Jahren eine Herausforderung dar. Ob dieses Verfahren geändert werden muss und wie dies geschehen könnte, wurde bisher überwiegend aus juristischer ${ }^{3}$ und politikberatender ${ }^{4}$ Perspektive diskutiert. Nachfolgend soll ein politikwissenschaftlicher Beitrag zu dieser Debatte geleistet werden.

\section{Der konstitutive Parlamentsvorbehalt in der Diskussion}

Der konstitutive Parlamentsvorbehalt ist Gegenstand intensiver wissenschaftlicher Beschäftigung, vor allem aus juristischer Perspektive. ${ }^{5}$ Die politikwissenschaftliche Analyseebene dieses Problems ist jedoch von der juristischen nicht zu trennen, wie Nina Philippi überzeugend darstellt. ${ }^{6}$ Auch Sebastian Harnisch hat die Veränderungen des Entscheidungsprozesses, die Beziehungen zwischen dem Deutschen Bundestag und der Bundesregierung und die Domestizierungstendenzen des Parlamentes in den Vordergrund seiner Arbeit gestellt. ${ }^{7}$ Das parlamentarische Entscheidungsverfahren wurde zuletzt von Rafael Biermann analysiert. ${ }^{8}$ Aus politikwissenschaftlicher Sicht hat sich Sven Bernhard Gareis mit den aktuellen Herausforderungen für den Parlamentsvorbehalt beschäftigt. ${ }^{9}$

\subsection{Die Staatspraxis}

Die endgültige Abstimmung über einen Auslandseinsatz der Bundeswehr im Plenum des Bundestages markiert das Ende eines langen Entscheidungsprozesses, an dem das Parlament, vor allem der Auswärtige und der Verteidigungsausschuss, mitwirken ${ }^{10}$, wobei das

3 Dieter Wiefelspütz, Die militärische Integration der Bundeswehr und der konstitutive Parlamentsvorbehalt, in: ZaöRV, Band 64 (2004), S. 363 - 389.

4 Vor allem Stephan Mair (Hrsg.), Auslandseinsätze der Bundeswehr. Leitfragen, Entscheidungsspielräume und Lehren, SWP-Studie 27, Berlin 2007.

5 Hier sind vor allem zu nennen: Dieter Wiefelspütz, Das Parlamentsheer, Berlin 2005; ders., Der konstitutive wehrverfassungsrechtliche Parlamentsbeschluss, in: ZParl, 38. Jg. (2007), H. 1, S. 3 - 16; Martin Limpert, Auslandseinsatz der Bundeswehr, Berlin 2002; Andreas Gilch, Das Parlamentsbeteiligungsgesetz. Die Auslandsentsendung der Bundeswehr und deren verfassungsrechtliche Gestaltung. Dissertation, Julius-Maximilians-Universität Würzburg 2005. Allgemeiner zu juristischen Aspekten der Internationalisierung der Streitkräfte Roman Schmidt-Radefeldt, Parlamentarische Kontrolle der internationalen Streitkräfteintegration, Berlin 2005.

6 Nina Philippi, Bundeswehr-Auslandseinsätze als außen- und sicherheitspolitisches Problem des geeinten Deutschland, Frankfurt am Main 1997.

7 Sebastian Harnisch, Internationale Politik und Verfassung. Die Domestizierung der deutschen Sicherheits- und Europapolitik, Baden-Baden 2006.

8 Rafael Biermann, Der Deutsche Bundestag und die Auslandseinsätze der Bundeswehr. Zur Gratwanderung zwischen exekutiver Prärogative und legislativer Mitwirkung, in: ZParl, 35. Jg (2004), H. 4, S. $607-626$.

9 Sven Bernhard Gareis, Bedingt bündnisfähig? Die parlamentarische Kontrolle internationaler Bundeswehreinsätze und die deutschen Verpflichtungen in NATO und EU, in: Gesellschaft Politik - Wirtschaft, H. 2/2007, S. 217 - 230.

10 Detailliert Florian Schröder, Das parlamentarische Zustimmungsverfahren zum Auslandseinsatz der Bundeswehr in der Praxis, Köln / Berlin / München 2005. 
formelle Verfahren im Parlamentsbeteiligungsgesetz (PBG) ${ }^{11}$ geregelt ist. Verfahrenstechnisch wird dem Bundestag ein Antrag auf konstitutiven Parlamentsbeschluss nach der Regierungsentscheidung zugeleitet. Nach dem ersten Durchgang im Plenum wird er in die Ausschüsse überwiesen, dabei ist der Auswärtige Ausschuss traditionell federführend, der Haushalts- und der Verteidigungsausschuss immer mitberatend; meistens wird die Vorlage auch vom Ausschuss für wirtschaftliche Zusammenarbeit und Entwicklung behandelt. Der Haushaltsausschuss beschäftigt sich ausschließlich mit den finanziellen Aspekten der Vorlage. Mit einer zweiten Beratung im Plenum wird über den Antrag abgestimmt. Seit 1994 findet traditionell immer eine namentliche Abstimmung statt - mit bisher zwei Ausnahmen. ${ }^{12}$

\subsection{Multinationale Verbände als Herausforderung für den Parlamentsvorbehalt}

Sowohl die NATO als auch die Europäische Union verfolgen seit einiger Zeit das Projekt multinationaler Verbände für rasche Einsätze in Konfliktgebieten. ${ }^{13}$ Einzelne Mitgliedstaaten sollen feste Truppenzusagen für einen bestimmten Zeitraum geben, damit die Kräfte in fünf bis sieben Tagen einsatzbereit sind. Angesichts dieser Herausforderung wird vorgeschlagen, die Entscheidungsprozedur im Bundestag zu verändern beziehungsweise zu modifizieren. ${ }^{14}$ So wurde schon im Koalitionsvertrag 2005 festgelegt: „Das Parlamentsbeteiligungsgesetz bleibt Verfahrensgrundlage der konstitutiven Entscheidung des Parlaments über Auslandseinsätze der Bundeswehr. Sollte sich im Lichte bisher gewonnener Erfahrungen ein Bedarf zur Weiterentwicklung ergeben, so werden die Koalitionsfraktionen Initiativen einbringen." 15

Die Befürworter einer solchen Veränderung (hauptsächlich aus den Reihen der CDU/ CSU) argumentieren mit der Notwendigkeit, erstens ein zeitliches Problem zu lösen, da die Abstimmung im Bundestag einen Militäreinsatz verzögern könnte und zweitens die Handlungsfähigkeit der EU beziehungsweise NATO aufrechtzuhalten, da im Falle einer Ablehnung durch den Bundestag die gesamte Sicherheitspolitik dieser Organisationen infrage gestellt werden könnte. ${ }^{16}$ Diese Argumentation zeigte sich schon in der Debatte um das Parlamentsbeteiligungsgesetz. ${ }^{17}$ Auch das Bundesverfassungsgericht sah 1994 in seinem Ur-

11 Gesetz über die parlamentarische Beteiligung bei der Entscheidung über den Einsatz bewaffneter Streitkräfte im Ausland vom 18. März 2005, BGBl I 2005, S. 775.

12 Es handelte sich um die nachträgliche Billigung der Rettungsoperation Libelle in Albanien im Jahre 1997 und um den humanitären Einsatz in Osttimor (INTERFET) im Oktober 1999.

$13 \mathrm{Zu}$ den European Battle Groups vgl. Gustav Lindstrom, Enter the EU Battlegroups, Challiot Paper No. 97, Institute for Security Studies, February 2007; zur NATO Response Force umfangreich Petr Zlatohlávek, NATO Response Force: úvod do problematiky, Studie informačního centra o NATO, svazek 2, Sdružení Jagello 2000, Praha / Ostrava 2007.

14 Für eine gute Übersicht der Argumente für eine Veränderung der Entscheidungsprozedur vgl. Timo Noetzel / Benjamin Schreer, Parlamentsvorbehalt auf dem Prüfstand, SWP-Aktuell 10, Februar 2007 beziehungsweise dies., Vernetzte Kontrolle: Zur Zukunft des Parlamentsvorbehalts, in: Stephan Mair (Hrsg.), a.a.O., S. 35 - 42.

15 Gemeinsam für Deutschland. Mit Mut und Menschlichkeit. Koalitionsvertrag von CDU, CSU und SPD, S. 154.

16 CDU will Soldaten schneller entsenden, in: FTD vom 24. Januar 2007; Timo Noetzel/ Benjamin Schreer, Parlamentsvorbehalt, a.a.O., S. 1 f.

17 Andreas Gilch, a.a.O, S. 207 f. 
teil die Möglichkeit vor, die „parlamentarische Beteiligung nach der Regelungsdichte abzustufen, in der die Art des möglichen Einsatzes der Streitkräfte bereits durch ein vertraglich geregeltes Programm militärischer Integration vorgezeichnet ist"18.

Die letzte öffentlich geführte politische Diskussion über den Parlamentsvorbehalt wurde unmittelbar dadurch initiiert, dass Deutschland 2006 zuerst für eine Eingreiftruppe (European Battle Group) und in der zweiten Hälfte des Jahres auch für die NATO Response Force Soldaten zur Verfügung stellte. ${ }^{19}$ Nach einem Vorstoß von Innenminister Wolfgang Schäuble im Dezember $2006^{20}$ sprach sich die CDU/CSU-Fraktion im Januar 2007 für einen Doppelbeschluss zu Auslandseinsätzen aus. Danach sollte der Bundestag am Anfang jeder Wahlperiode einen „Vorratsbeschluss“ fassen. In jedem konkreten Einsatzfall wäre danach auch ein Beschluss notwendig, allerdings ohne eine Obergrenze, wie viele Soldaten eingesetzt werden können. ${ }^{21}$

Die Gegner einer solchen Veränderung bestreiten in erster Linie das Zeitproblem. Die Reaktionskräfte sollen in fünf bis sieben Tagen einsatzbereit sein - in dieser Zeit könne auch der Bundestag einen Beschluss fassen. In der Vergangenheit sei es nie zu Verzögerungen wegen der Abstimmung im Bundestag gekommen. Das sicherheitspolitische Problem könne durch eine frühe Einbindung des Parlaments in den Entscheidungsprozess gelöst werden. $\mathrm{Zu}$ diesem Zwecke wäre zum Beispiel ein ähnliches Abkommen möglich, welches bereits zwischen Bundestag und Bundesregierung bezüglich der Angelegenheiten der Europäischen Union besteht. ${ }^{22}$

Die politische Diskussion Anfang 2007 endete im Widerstand der SPD-Fraktion ${ }^{23}$ und der Errichtung einer Arbeitsgruppe der Koalitionsfraktionen ${ }^{24}$, die jedoch offensichtlich zu keiner Einigung gekommen ist. Auf der politikwissenschaftlichen und politikberatenden Ebene wird jedoch weiter diskutiert. ${ }^{25}$ Im Sinne der Forderung von Rafael Biermann nach mehr Fallstudien ${ }^{26}$ wird im Folgenden der Entscheidungsprozess um den Einsatz der Bundeswehr im Kongo im Jahre 2006 analysiert und gefragt, ob die Erfahrungen wirklich für eine Veränderung der Entscheidungsregeln sprechen. ${ }^{27}$

18 BVerfGE 90, S. 286 (S. 388).

19 In der ersten Jahreshälfte 2006 handelte es sich um eine Eingreiftruppe, die aus 1.500 deutschen Soldaten und vier Franzosen bestand, in der zweiten Jahreshälfte beteiligte sich Deutschland an der 7. Rotation der NATO Response Force mit rund 6.700 Soldaten, darunter 3.300 Heeressoldaten (Eurokorps).

20 Vgl. SPD kritisiert Schäuble, in: FAZ vom 13. Dezember 2006.

21 Vgl. CDU will Soldaten schneller entsenden, in: FTD vom 24. Januar 2007.

22 Vgl. Rainer Stinner, Bleibt es bei der Parlamentsarmee?, in: FAZ vom 15. September 2006.

23 Lediglich der Fraktionsvorsitzende und ehemalige Verteidigungsminister Peter Struck hat sich für eine „weniger detaillierte“ Beschlussfassung des Bundestages ausgesprochen. Dies wurde jedoch nur als ein „Test“ der eigenen Fraktion empfunden. Vgl. Struck testet eigene Fraktion, in: Frankfurter Rundschau vom 22. Februar 2007; SPD-Fraktionschef für Neuregelung von Auslandseinsätzen, in: Die Welt vom 22. Februar 2007.

24 Vgl. Steinmeier begräbt CDU-Plan für schnellen Militäreinsatz, in: FTD vom 30. Januar 2007.

25 Vgl. vor allem Stefan Mair (Hrsg.), a.a.O.

26 Rafael Biermann, a.a.O., S. 620.

27 Der Fall des Kongo-Einsatzes wird als Argument für eine Veränderung der Entscheidungsprozedur benutzt. Vgl. Timo Noetzel / Benjamin Schreer, Parlamentsvorbehalt, a.a.O., S. 1. 


\section{Der Entscheidungsprozess für den Einsatz der Bundeswehr in der Demokratischen Republik Kongo 28}

\subsection{Beginn des Entscheidungsprozesses}

Am 27. Dezember 2005 ersuchten die Vereinten Nationen (UNO) beziehungsweise der Leiter der Abteilung für Friedensoperationen Jean-Marie Guéhenno offiziell die EU, die bestehende Friedensmission der UNO in Kongo (MONUC) durch eine multinationale Truppe zu verstärken, um die damals noch für den 29. April 2006 geplanten Wahlen abzusichern. ${ }^{29}$

Die EU befand sich im Rahmen der Europäischen Sicherheits- und Verteidigungspolitik seit Januar 2005 in der so genannten Initial Operational Capability und verfügte somit mindestens über eine einsatzbereite Eingreiftruppe. ${ }^{30}$ In der ersten Jahreshälfte 2006 waren zwei einsatzbereit: eine unter der Führung von Spanien mit Truppenteilen aus Griechenland, Italien und Portugal und eine, die aus Bundeswehrverbänden bestand. ${ }^{31}$ Ein Einsatz der deutschen Soldaten schien schon in den ersten politischen Diskussionen im Rahmen der EU, die am 10. Januar 2006 stattfanden, der einfachste Weg zu sein, der Anfrage der Vereinten Nationen zu entsprechen, da der Verband unter spanischer Führung vorrangig für amphibische Operationen ausgestattet und organisiert war. ${ }^{32}$

In die öffentliche Debatte in Deutschland gelangte das Thema am 16. Januar 2006, nachdem Medienvertreter durch einen Sprecher des Bundesministeriums der Verteidigung (BMVg) über die geprüfte Anfrage der UNO informiert worden waren. ${ }^{33} \mathrm{Zu}$ diesem Zeitpunkt war von einem möglichen Einsatz der deutschen Eingreiftruppe die Rede. ${ }^{34}$ Am 18. Januar informierte die Bundesregierung in Ausschusssitzungen die Abgeordneten über die Abläufe und Sondierungsgespräche in der EU, deren Ausgang vor allem hinsichtlich einer möglichen Beteiligung deutscher Truppen als völlig ergebnisoffen präsentiert wurde. ${ }^{35}$

28 Das Folgende basiert unter anderem auf mehreren Hintergrundgesprächen und strukturierten Interviews zum Parlamentsvorbehalt sowie zum Kongo-Einsatz der Bundeswehr, die der Verfasser im Juli 2006 und September 2007 sowohl mit einigen Mitgliedern des Verteidigungsausschusses als auch mit Fachreferenten sämtlicher Fraktionen des Bundestages geführt hat. Auf einzelne Gespräche wird nicht konkret verwiesen.

29 Vgl. Antrag der Bundesregierung: Beteiligung deutscher Streitkräfte an der EU-geführten Operation RD Congo zur zeitlich befristeten Unterstützung der Friedensmission MONUC der Vereinten Nationen während des Wahlprozesses in der Demokratischen Republik Kongo auf Grundlage der Resolution 1671 (2006) des Sicherheitsrates der Vereinten Nationen vom 25. April 2006, BTDrs. 16/1507 (weiterhin BT-Drs. 16/1507); vgl. auch Denis M. Tull, Die Führung und Beteiligung der Bundeswehr an EUFOR RD Congo, in: Stephan Mair (Hrsg.), a.a.O., S. 68 - 77, S. 68.

30 Gustav Lindstrom, a.a.O., S. 20.

31 Gustav Lindstrom, a.a.O., S. 57. Es handelte sich um Einheiten der Luftlandebrigade 26 „Saarland" mit französischen Verbindungsoffizieren (insgesamt 1.500 deutsche und vier französische Soldaten).

32 Gustav Lindstrom, a.a.O., S. 58, Fn. 126.

33 Vgl. Mission im Land der Mörderbanden, in: SZ vom 16. Januar 2006; Bundeswehr prüft Kongo-Einsatz, in: Der Spiegel vom 16. Januar 2006.

34 Vgl. EU prüft Kongo-Einsatz, in: FAZ vom 17. Januar 2006.

35 Vgl. Jung hält Einsatz der Bundeswehr im Kongo für möglich, in: Die Welt vom 23. Januar 2006. 
Dies bildete den Anfang des Meinungsbildungsprozesses im Parlament. Sowohl in der Sitzung des Verteidigungsausschusses als auch in den folgenden Tagen äußerten vor allem Verteidigungsexperten sämtlicher Fraktionen Einwände gegen den möglichen Einsatz der deutschen Eingreiftruppe, und die allgemeine Haltung von Regierung und Parlament wurde als skeptisch bezeichnet. ${ }^{36}$

\subsection{Die Entscheidung für den Einsatz}

In einem Interview in der Bild am Sonntag vom 22. Januar 2006 sprach Verteidigungsminister Franz-Josef Jung von einer "gemeinsamen Verantwortung“ der EU gegenüber Afrika und schloss einen Bundeswehreinsatz zur Absicherung der Wahlen nicht aus. ${ }^{37}$ Dies wurde allgemein als Befürwortung eines Kongo-Einsatzes der Bundeswehr und somit als Abweichung von der bisher zurückhaltenden Position der Regierung und der Bundeskanzlerin empfunden. ${ }^{38}$ Die Exekutive hatte offensichtlich erkannt, dass man sich einem Einsatz der Bundeswehr bei einer gemeinsamen EU-Aktion nicht völlig entziehen könne, aber entschieden, dass eine Entsendung einer rein deutschen Truppe nicht in Frage komme. ${ }^{39}$ Bei einem Gipfeltreffen zwischen Bundeskanzlerin Angela Merkel und dem französischen Präsidenten Jacques Chirac am 24. Januar in Versailles und bei den parallelen Verhandlungen in Brüssel wurde diese Position vorgetragen und der Einsatz von „1500 Deutschen und vier Franzosen“ ausgeschlossen. ${ }^{40}$ Über diese Entscheidung wurde der Bundestag am 25. Januar in den Sitzungen des Auswärtigen und des Verteidigungsausschusses umfassend und früher als die Presse unterrichtet. Verteidigungsminister Jung betonte jedoch noch im Verteidigungsausschuss, dass erstens über einen Einsatz von Soldaten nicht definitiv entschieden sei, sondern es sich auch um einen Polizeieinsatz handeln könnte, und zweitens Deutschland nicht in die Lage geraten wolle, eine Führungsposition im Kongo übernehmen zu müssen. Die Bedenken einzelner Abgeordneter zu möglichen Zusammenstößen mit Kindersoldaten, zu der Finanzierung oder zu den Fähigkeiten und Kapazitäten der Bundeswehr wurden von der Regierung größtenteils ausdrücklich geteilt.

Erst nachdem der Bundestag informiert worden war, wurde auch in der Presse bekannt gegeben, dass die Eingreiftruppe nicht eingesetzt werden solle. ${ }^{41}$ Für den „Nicht-Einsatz“ gab es neben innerdeutschen Vorbehalten auch stichhaltige militärische Gründe. ${ }^{42}$ Erstens war die deutsche Truppe vor allem für Evakuierungseinsätze vorbereitet und ausgestattet, mit einer maximalen Einsatzdauer von sieben Tagen. ${ }^{43}$ Zweitens entsprach die geplante Absicherung der Wahlen und die Abschreckungsfunktion nicht dem Profil eines Kampf-

36 Vgl. Bundeswehr soll nicht alleine in den Kongo, in: Berliner Zeitung vom 19. Januar 2006; Berlin beurteilt einen Einsatz der Bundeswehr in Kongo skeptisch, in: Handelsblatt vom 19. Januar 2006; Bundeswehr soll in den Kongo, in: SZ vom 21. Januar 2006.

37 Militärische Drohkulisse gegen den Iran nötig, in: Bild am Sonntag vom 22. Januar 2006.

38 Vgl. Jung hält Einsatz der Bundeswehr in Kongo für möglich, a.a.O.

39 Diese Verhandlungsposition der Regierung ist bereits nachzulesen in: Abwehr in Versailles, in: Der Spiegel vom 23. Januar 2006.

40 Vgl. auch Denis M. Tull, a.a.O., S. 69.

41 Vgl. Merkel verhindert Kongo-Einsatz der EU-Truppe, in: Berliner Zeitung vom 26. Januar 2006; Keine Führungsrolle bei Kongo-Einsatz, in: FAZ vom 28. Januar 2006.

42 Vgl. Gustav Lindstrom, a.a.O., S. 57 f.

43 Vgl. Anders als der Balkan, in: FAZ vom 24. Januar 2006. 
gruppeneinsatzes, das heißt einem raschen Einsatz zur Konfliktlösung. Drittens war der geplante Einsatz zu lang und deckte sich nicht mit dem zeitlichen Ablauf, in dem die einzelnen Eingreiftruppen einsatzbereit waren. ${ }^{44}$ Ausschlaggebend für die deutsche Debatte und die Ablehnung des Einsatzes deutscher Soldaten waren jedoch eindeutig politische Gründe: die fehlende Bereitschaft, in einem traditionell frankophonen und für die Bundeswehr neuen Gebiet eine riskante Militäroperation zu führen und die Vermutung, dass die französische Regierung hinter dem UNO-Gesuch stehe, um deutsche Truppen für französische Interessen zu verwenden. ${ }^{45}$ Die Bundesrepublik konnte sich jedoch der gesamteuropäischen Verantwortung für einen ruhigen Ablauf der kongolesischen Wahlen und somit für die Stabilität im Kongo nicht ganz entziehen.

Zwischen Bundesregierung und Bundestag bestand Einvernehmen, dass die Verantwortlichkeit für den Kongo-Einsatz gemeinsam bei allen beteiligten europäischen Ländern liegen müsse. Deshalb stellte der Verteidigungsminister bei den internationalen Verhandlungen höchstens eine Beteiligung deutscher Truppen an einem von einer anderen Nation geführten multinationalen EU-Verband in Aussicht. Nach der grundsätzlichen Entscheidung zwischen Deutschland und Frankreich, dass eine solche EU-Truppe im Kongo eingesetzt werden solle, wurde der Entscheidungsprozess innerhalb der EU fortgesetzt und eine Erkundungsmission unter der Leitung des deutschen Brigadegenerals Heinrich Brauß in seiner Funktion als Leiter der zivil-militärischen Zelle im EU-Militärstab in den Kongo entsandt.

$\mathrm{Zu}$ einer reservierten bis ablehnenden Einstellung bei den Abgeordneten beider Regierungsfraktionen trug die Reise einzelner Parlamentarier in den Kongo in der ersten Februarwoche bei. So warnte zum Beispiel Anita Schäfer (CDU/CSU), Mitglied im Verteidigungsausschuss, nach ihrer Rückkehr vor einer möglichen Überforderung der Bundeswehr im Kongo und berichtete über divergierende Meinungen in der kongolesischen Regierung, ob ein Einsatz internationaler Truppen überhaupt gewünscht sei. ${ }^{46}$

In der folgenden Sitzung des Verteidigungsausschusses am 8. Februar stellte Bundesverteidigungsminister Jung verschiedene Einsatzoptionen für die Bundeswehr je nach Truppenstärke und -zusammensetzung sowie Stationierung ganz oder nur teilweise im Kongo vor, die bis zu dem Zeitpunkt diskutiert worden waren. Der Nachricht, dass ein Bundeswehrkontingent unter Umständen bis zu 500 Soldaten umfassen könnte, wurde in den Medien viel Aufmerksamkeit gewidmet, und auch die Informationen über den Standpunkt der Bundesregierung hinsichtlich des Kongo-Einsatzes, den der Verteidigungsminister am 8. Februar dem Verteidigungsausschuss vorgetragen hatte, fanden sehr schnell ihren Weg in die Presse. ${ }^{47}$

Im Laufe des Februars 2006 wurden auf der Basis des Berichts des EU-Erkundungsteams verschiedene Einsatzoptionen erwogen und im Politischen und Sicherheitspolitischen Komitee (PSK) und Militärausschuss der EU ein so genanntes Optionspapier erarbeitet. ${ }^{48}$

44 Gustav Lindstrom, a.a.O., S. 58.

45 Vgl. Fallschirmjäger nach Kinshasa, in: Der Spiegel vom 30. Januar 2006.

46 Vgl. Widerstand gegen Einsatz der Bundeswehr im Kongo, in: Die Welt vom 7. Februar 2006.

47 Vgl. Bundeswehr nimmt Kurs auf Kongo, in: Berliner Zeitung vom 9. Februar 2006; Deutschland schickt Soldaten in den Kongo, in: Die Welt vom 10. Februar 2006.

48 Vgl. Drei Optionen für den Kongo, in: SZ vom 14. Februar 2006; EU berät über Kongo-Einsatz, in: FAZ vom 14. Februar 2006. 
Zum Verhältnis zwischen Bundesregierung und Bundestag sei erwähnt, dass den Abgeordneten zunächst die Einsicht in dieses Dokument verweigert, nach einer direkten Forderung in der Ausschusssitzung jedoch ermöglicht wurde.

Die endgültige Entscheidung auf der europäischen Ebene verzögerte sich jedoch, da die Anforderungen an den Militäreinsatz zu vage waren und keiner der EU-Staaten bereit war, die Operation zu führen. ${ }^{49}$ In Bezug auf die deutsche Beteiligung wurde Druck aus dem Bundestag ausgeübt, dass im Kongo keine deutschen Kampftruppen eingesetzt werden sollten. Laut den Äußerungen einiger Abgeordneter hätte ein solches Vorhaben im Bundestag keine Chance gehabt, gebilligt zu werden. ${ }^{50}$

\subsection{Sieben Kriterien für den Einsatz}

Nach einer informellen Tagung der EU-Verteidigungsminister in Innsbruck am 6. und 7. März 2006 wurde in Deutschland der Sinn des Einsatzes noch stärker bezweifelt, und zwar auch von den Verteidigungsexperten beider Koalitionsfraktionen. ${ }^{51}$ Als Reaktion auf den wachsenden internationalen Druck auf Deutschland, die Führung der Mission zu übernehmen einerseits und die deutlichen innenpolitischen Vorbehalte andererseits stellte die Exekutive für die nächsten Verhandlungen einige Bedingungen, an die eine deutsche Beteiligung geknüpft werden sollte: Zustimmung der kongolesischen Regierung; ein Mandat der Vereinten Nationen; klar abgegrenztes Aufgabenprofil; Absicherung der Wahlen; Begrenzung des Einsatzes auf den Wahlzeitraum; mögliches Einsatzgebiet im Kongo im Raum Kinshasa und substantielle Beteiligung durch mehrere EU-Partner. ${ }^{52}$ Auf der Sitzung des deutsch-französischen Ministerrates am 14. März wurde im Wesentlichen vereinbart, dass Deutschland und Frankreich je ein Drittel der Truppen stellen und Frankreich das Kommando im Feld (Field Headquarters) übernehmen sollte. Dies implizierte die Übernahme der Gesamtleitung der Operation durch Deutschland (Operational Headquarters) und somit die Führung und Koordination des Einsatzes - eine Rolle, in die Deutschland seit Anfang der Verhandlungen im Januar 2006 nicht hineinmanövriert werden wollte. ${ }^{53}$

$\mathrm{Zu}$ der Notwendigkeit, die genannten Kriterien zu formulieren und mit dem Parlament abzustimmen, trug eine weitere parlamentarische Aktivität bei. Der Verteidigungsausschuss hatte sich bei seiner auswärtigen Tagung in Brüssel am 8. März selbst ein Bild über die Lage verschaffen wollen. Nach Gesprächen mit dem Hohen Repräsentanten der EU, Javier Sola$n a$, und Vertretern des EU-Militärausschusses zeigten sich einige Abgeordnete enttäuscht

49 Vgl. Schwierige Diskussionen, in: FAZ vom 22. Februar 2006.

50 Vgl. EU sucht Führung für Kongo-Mission, in: Handelsblatt vom 13. Februar 2006; Vorbehalte gegen Kongo-Einsatz, in: FAZ vom 16. Februar 2006.

51 Vgl. die Äußerungen von Thomas Kossendey und Hans-Peter Barthels in: Bundestag bremst bei Kongo-Einsatz, in: Handelsblatt vom 8. März 2006.

52 Die erste Version der Bedingungen ist den anderen europäischen Staaten offensichtlich auf dem Treffen der EU-Verteidigungsminister in Innsbruck am 6./7. März mitgeteilt worden, vgl. FDP kritisiert Kongo-Einsatz, in: SZ vom 9. März 2006; Koalition unter Bedingungen zu KongoEinsatz bereit, in: FAZ vom 10. März 2006.

$53 \mathrm{Zu}$ dem Verhandlungsprozess auf internationaler Ebene vgl. Peter Schmidt, Nationale Entscheidungsspielräume in der Europäischen Union und Vereinten Nationen, in: Stephan Mair (Hrsg.), a.a.O., S. 50 - 58, S. 56. 
über den Stand der Einsatzvorbereitungen und ließen ihren Unmut in der Presse verlauten. ${ }^{54}$ Besonders die Abgeordneten der Fraktionen CDU/CSU, FDP und Die Linke waren unzufrieden mit den ihrer Ansicht nach vagen Aussagen von Solana zum genauen Aufgabenspektrum des Militärs im Kongo, bezüglich der erforderlichen Zahl von Soldaten und der ungeklärten Stellung der kongolesischen Regierung. Die Abgeordneten der SPD hielten dies hingegen nicht für besorgniserregend. Alle im Bundestag vertretenen Parteien teilten zu diesem Zeitpunkt jedoch die Ansicht, dass die Bundesregierung bis dato kein zustimmungsfähiges Einsatzkonzept vorgelegt hatte. ${ }^{55}$ Allerdings äußerten sich die Vertreter der FDP, der CDU und vor allem der CSU zurückhaltend; der Einsatz wurde eher von der SPD und den Grünen befürwortet. ${ }^{56}$

Nach der Übereinkunft zwischen Frankreich und Deutschland vom 14. März wurde der Bundestag am 16. März offiziell auf einer gemeinsamen Sitzung des Auswärtigen und Verteidigungsausschusses durch den Außen- und den Verteidigungsminister informiert. Zwischen diesen beiden Tagen (vor allem am Abend und in der Nacht vom 14. auf den 15. März) wurden informell die Obleute der Regierungsfraktionen im Auswärtigen beziehungsweise Verteidigungsausschuss in Kenntnis gesetzt und die bestehenden Bedenken einiger Parlamentarier der Koalitionsfraktionen durch eine Reihe informeller Gespräche vorübergehend gedämpft, so dass die Pressemeldungen nach der gemeinsamen Sitzung überwiegend von einer Billigung des Kongo-Einsatzes im Bundestag ausgingen. ${ }^{57} \mathrm{Um}$ der innenpolitischen Kritik am Ergebnis der Übereinkunft mit Frankreich auszuweichen, übte die Bundesregierung öffentlichen Druck auf Javier Solana aus und forderte die Erfüllung der oben genannten Bedingungen, insbesondere die Zustimmung der kongolesischen Regierung. ${ }^{58}$ Die kritische Haltung der Parlamentarier, vor allem der Unionsfraktion, kam jedoch in den folgenden Tagen erneut zum Ausdruck. ${ }^{59}$ Auch die Freidemokraten nahmen eine ablehnende Haltung ein. Der Beschluss ihres Parteipräsidiums zum Kongo-Einsatz ist auch deshalb interessant, weil die FDP ablehnte, dass „die Bundesregierung vor einer Befassung des Bundestages einseitig internationale Zusagen gibt“. Dies widerspreche der Verfassung, da die Bundeswehr eine Parlamentsarmee sei. ${ }^{60}$ Die FDP profilierte sich auch im weiteren Ablauf als „Hüterin“ der Rechte des Bundestages in diesem Entscheidungspro-

54 Vgl. Weiter Bedenken wegen Kongo, in: FAZ vom 9. März 2006; Für Kongo-Einsatz der EU fehlt Planung, in: Die Welt vom 10. März 2007; Koalition unter Bedingungen zu Kongo-Einsatz bereit, in: FAZ vom 10. März 2006.

55 Vgl. Siebert stellt EU-Einsatz im Kongo in Frage, in: SZ vom 14. März 2006; Klares Jein zum Kongo-Einsatz, in: SZ vom 16. März 2006.

56 Vgl. Kongo-Mission entzweit Bundestag, in: Frankfurter Rundschau, 15. März 2006.

57 Vgl. Jung setzt Solana eine Frist, in: FAZ vom 17. März 2006; Berlin für Einsatz der Bundeswehr in Kongo, in: Frankfurter Rundschau vom 17. März 2006; Einsatz im Kongo so gut wie perfekt, in: Berliner Zeitung vom 17. März 2006.

58 Vgl. Solana spricht mit Kabila über Kongo-Einsatz, in: SZ vom 18. März 2006; Jung setzt Solana eine Frist, a.a.O.

59 Kritik aus der Koalition und der Bundeswehr an Kongo-Einsatz, in: FAZ vom 20. März 2006; Solana verhandelt mit Kabila; in: SZ vom 20. März 2006; FDP und CSU wollen nicht in den Kongo, in: Die Welt vom 21. März 2006.

60 FDP-Präsidium lehnt Kongo-Einsatz unter gegenwärtigen Umständen ab, Pressemitteilung der FDP, 20. März 2006, http://www.liberale.de/files/593/FDK7906_Kongo.pdf (Zugriff am 10. Oktober 2007). 
zess. ${ }^{61}$ Zudem warf ihre verteidigungspolitische Sprecherin Birgit Homburger dem Verteidigungsminister Wortbruch vor in der Frage, welcher Staat den Einsatz führen werde.

Nach dem 20. März wurden weitere Bedingungen der Bundesregierung erfüllt: die Zustimmung der kongolesischen Regierung und Truppenzusagen weiterer EU-Staaten. ${ }^{62}$ Somit rückte der Einsatz immer näher. ${ }^{63}$ Die Kabinettsentscheidung über den Einsatz wurde auf Ende März verschoben, da man Beschlüsse im Rahmen der UNO und EU abwartete. Wegen der Osterpause verzögerte sich die formelle Entscheidung im Bundestag bis Mai 2006. ${ }^{64}$ Um die Zustimmung des Parlaments warb die Bundeskanzlerin am Montag, den 27. März, bei einem Treffen mit den Vorsitzenden aller Bundestagsfraktionen. ${ }^{65}$ Im April 2006 wurden vor allem militärische Einzelheiten des Einsatzes ausgearbeitet und international verhandelt. Zugleich verschob die kongolesische Regierung den Wahltermin aus organisatorischen Gründen auf Juli 2006. Am 18. April brachen Elke Hoff (FDP), Winfried Nachtwei und Hans-Christian Ströbele (Die Grünen) in den Kongo auf, um sich ein Bild über die Lage zu verschaffen. ${ }^{66}$ Diese Reise führte zur Bestätigung in ihren Haltungen, also einerseits ablehnend (Elke Hoff, Hans-Christian Ströbele), andererseits befürwortend (Winfried Nachtwei). ${ }^{67}$

Am 25. April 2006 verabschiedete der UN-Sicherheitsrat die völkerrechtliche Grundlage für einen Militäreinsatz, die Resolution 1671 (2006). Daraufhin startete am 27. April die EU offiziell eine gemeinsame Aktion und benannte den deutschen General Karlheinz Viereck zum Operationskommandeur. Die folgende Debatte in Deutschland drehte sich vor allem um die Frage, ob das Einsatzgebiet der deutschen Soldaten im ganzen Kongo oder nur in Kinshasa sein sollte (wie es auch später im Beschluss festgelegt wurde), und um die gestiegenen Einsatzkosten. Mit der ersten Frage befasste sich vor allem der Verteidigungsausschuss, zu den Kosten wurde am 11. Mai der Verteidigungsminister in den Haushaltsausschuss zitiert. Nach einer weiteren Verschiebung des Wahltermins im Kongo auf den 30. Juli wurde auch die Kabinettsentscheidung auf den 17. Mai vertagt. Vor allem die Parlamentarier der FDP kritisierten in der Zwischenzeit das unklare Einsatzkonzept. In der Fraktion der SPD sorgte der Vorschlag ihres verteidigungspolitischen Sprechers Rainer Arnold, das Einsatzgebiet auf den ganzen Kongo auszuweiten, für Unmut. ${ }^{68}$

61 Vgl. Schwere Vorwürfe gegen Jung, in: Die Welt vom 22. März 2006; Bundestag hat keine Entscheidungsfreiheit, in: Die Welt vom 25. März 2006; Merkel erläutert Kongo-Pläne, in: SZ vom 28. März 2006.

62 Vgl. Kongo-Einsatz rückt näher, in: FAZ vom 21. März 2006; Einen wichtigen Schritt weiter gekommen, in: Der Tagesspiegel vom 21. März 2006.

63 Vgl. Ohne Plan in den Kongo, in: Die Welt vom 21. März 2006.

64 Die Sitzungswochen des Bundestages: 27. bis 31. März, 3. bis 7. April, 8. bis 11. Mai 2006. Vgl. Bundeswehr-Mandat für den Kongo kommt Anfang Mai, in: Die Welt vom 23. März 2006; Bundeswehrsoldaten sollen Ende Mai in Kongo sein, in: FAZ vom 23. März 2006; Entscheidung zu Kongo später, in: Frankfurter Rundschau vom 23. März 2006.

65 Vgl. Merkels Werben um Einsatz in Kongo überzeugt Grüne, in: FTD vom 28. März 2006; Merkel erläutert Kongo-Pläne, in: SZ vom 28. März 2006; Ausgeweitetes Kongo-Mandat?, in: FAZ vom 28. März 2006.

66 Vgl. Erkundungsmission im Kongo, in: SZ vom 18. April 2006.

67 Vgl. Kongo-Einsatz in der Kritik, in: SZ vom 22. April 2006; Die Erwartungen sind ungeheuer hoch, Interview mit Hans-Christian Ströbele, in: taz vom 22. April 2006.

68 Vgl. Widerstand in der SPD gegen größeren Kongo-Einsatz, in: Die Welt vom 4. Mai 2006. 


\subsection{Billigung der Regierungsvorlage im Bundestag}

Als die Bundesregierung den Einsatz am 17. Mai beschloss, war eine breite Zustimmung unter den Abgeordneten schon gesichert. Die intensiven Diskussionen der vergangenen Monate erklärte die Vorsitzende des Verteidigungsausschusses Ulrike Merten (SPD) in einem Interview damit, dass den Abgeordneten ,in durchaus guter Informationsabsicht schon in einem sehr frühen Stadium sehr breit Zahlen und Größenordnungen genannt wurden, als man überhaupt nicht absehen konnte, was der Einsatz als solcher erfordert" ${ }^{\text {" } 9}$.

Die Regierungsvorlage wurde dem Bundestag zugeleitet und nach der ersten Lesung am 19. Mai in die Ausschüsse überwiesen. Die Ergebnisse der Beratungen entsprachen den angekündigten Positionen aller Fraktionen: Im federführenden Auswärtigen Ausschuss wurde die Vorlage mit den Stimmen der Fraktionen CDU/CSU, SPD und Bündnis 90/Die Grünen gegen die Stimmen der Fraktionen FDP und Die Linke angenommen, ebenso im Rechtsausschuss und im Ausschuss für wirtschaftliche Zusammenarbeit und Entwicklung. Bei der Abstimmung im Ausschuss für Menschenrechte und humanitäre Hilfe war der Vertreter der Fraktion Die Linke abwesend. Im Verteidigungsausschuss gaben die beiden Abgeordneten der Fraktion Bündnis 90/Die Grünen unterschiedliche Voten ab. Im Haushaltsausschuss enthielten sich zwei Abgeordnete der SPD der Stimme, einer lehnte die Regierungsvorlage ab.

Am 1. Juni wurde über die Vorlage in zweiter Lesung im Plenum des Bundestages abgestimmt. In der anderthalbstündigen Plenardebatte wurde von den Befürwortern des Einsatzes noch einmal die Notwendigkeit der Stabilisierung des Kongo und der Glaubwürdigkeit der Vereinten Nationen sowie der Europäischen Sicherheits- und Verteidigungspolitik hervorgehoben. Die Redner der FDP führten als Grund für ihre ablehnende Haltung die schlechte militärische wie auch politische Vorbereitung des Einsatzes an und vor allem die Tatsache, dass die gesteckten Ziele mit dem Einsatz nicht erreicht werden könnten. Bei der Fraktion der Linkspartei basierte die Ablehnung auf Zweifeln an einer Konfliktlösung mit militärischen Mitteln und dem Verdacht, bei dem Einsatz gehe es lediglich um kongolesische Rohstoffe. ${ }^{70}$

An der Abstimmung nahmen 581 Abgeordnete teil: 440 stimmten mit Ja, 135 mit Nein, sechs enthielten sich der Stimme. ${ }^{71}$ Mit 94,63 Prozent aller Abgeordneten entsprach diese Beteiligung der traditionell hohen Teilnahme an namentlichen Abstimmungen über Auslandseinsätze der Bundeswehr. Mit 23,24 Prozent Gegenstimmen hatte der Kongo-Einsatz jedoch eine der größten Widerstandsquoten bei solchen Abstimmungen seit $1994 .{ }^{72}$ Ungewöhnlich groß (6,71 Prozent) war auch der Anteil der Abgeordneten, die abweichend von der Mehrheit ihrer Fraktion votierten. Insgesamt handelte es sich um 39 Abgeordnete,

69 Es wird eine breite Zustimmung geben, in: Saarbrücker Zeitung vom 19. Mai 2006.

70 Vgl. Plenarprotokoll der 37. Sitzung des Deutschen Bundestages, Berlin, 1. Juni 2006, S. 3236 ff.

71 Ebenda, S. 3259.

72 Mit Ausnahme der Abstimmung über den Bodeneinsatz in Bosnien und Herzegowina im Juni 1995 (Operation Determined Effort) und über den OEF-Einsatz im November 2001, wo jedoch das Abstimmungsverhalten durch die Verknüpfung mit der Vertrauensfrage stark beeinflusst war. Die Zahlen beziehen sich auf sämtliche Entscheidungen über neue Auslandseinsätze in den Jahren 1992 bis 2007. Die Datenerhebung ist Teil des Dissertationsprojekts des Autors. Es ist darauf hinzuweisen, dass die Mehrheiten im Bundestag für die Einsätze im Libanon 2006 und der Tornado-Flugzeuge in Afghanistan 2007 noch schmaler waren. 
überwiegend aus den Fraktionen der SPD und Bündnis 90/Die Grünen. In den bisherigen Abstimmungen waren es durchschnittlich circa 23 Abgeordnete, die sich der Fraktionsdisziplin nicht unterwarfen.

\section{Bewertung der Beteiligung des Deutschen Bundestages am Entscheidungsprozess}

Der Verlauf des Entscheidungsprozesses und die Kommunikation zwischen Regierung und Parlament über den Kongo-Einsatz wurden von den beteiligten Parlamentariern überwiegend negativ bewertet. ${ }^{73}$ Erstens gab es wechselnde Informationen hinsichtlich der Truppenstärke, der Einsatzdauer, der Beteiligung anderer europäischer Staaten und nicht zuletzt der Einsatzkosten. Dies war größtenteils dadurch bedingt, dass sich die Bundesregierung gleichzeitig in einem offenen Entscheidungsprozess innerhalb der EU befand. Im Falle des Kongo-Einsatzes ist eine relativ große Informationsoffenheit der Bundesregierung zu erkennen. Abgesehen davon, dass die Abgeordneten über die Anfrage der UNO im Januar 2006 aus der Presse erfuhren, wurden die zuständigen Ausschüsse rechtzeitig und umfassend informiert, vor allem hinsichtlich des Zögerns, die Rolle der „Führungsnation“ zu übernehmen. Auch über die Ergebnisse des deutsch-französischen Gipfeltreffens am 24. Januar wurden die Ausschüsse schon am übernächsten Tag beziehungsweise einige Obleute in Ausschüssen bereits am Abend desselben Tages informiert. Erst als es später um die Einsatzmodalitäten ging (vor allem um die Truppenstärke und die Beschränkung des Einsatzgebietes auf den Raum Kinshasa), gab sich der Verteidigungsminister verschlossener. So wich er in der Sitzung des Verteidigungsausschusses am 10. Mai der Frage nach der genaueren Soldatenzahl aus und fügte hinzu, man werde von ihm doch wohl keine Zahlen zum Kongo erwarten, denn er habe auch dazugelernt. ${ }^{74}$ Die genaue Truppenstärke wurde im Bundestag erst bekannt, nachdem die Bundesregierung den Einsatz am 17. Mai beschlossen hatte.

Zweitens glaubten einige Abgeordnete, Deutschland sei vor allem durch Frankreich in den Einsatz und in die Führungsrolle hineinmanövriert worden. ${ }^{75}$ Eine zu schnell signalisierte grundsätzliche Bereitschaft, die Anfrage der UNO positiv zu beantworten und sich am Einsatz zu beteiligen, wurde öffentlich von der FDP kritisiert. Diese Auffassung wurde auch von einigen CDU/CSU-Abgeordneten geteilt, die im Verlauf des ganzen Entscheidungsprozesses auf mehr Zurückhaltung drängten.

Vor allem der zweite Punkt demonstriert das eingangs aufgeworfene grundsätzliche Problem, wie manövrierfähig die Regierungspolitik zwischen Forderungen der Bündnispartner und innenpolitischen Beschränkungen ist. In der Tradition deutscher Sicherheitspolitik

73 Diese Behauptung stützt sich auf mehrere Interviews mit Abgeordneten aller Fraktionen. Die schärfste Äußerung im Hinblick auf den Entscheidungsprozess war, die Bundesregierung habe alle Fehler gemacht, die man nur machen konnte. Zu erwähnen ist auch die Äußerung des Vorsitzenden der CSU-Landesgruppe, Peter Ramsauer, der einige Monate später in der Diskussion über einen möglichen Bundeswehreinsatz in Sudan sagte, er mache Vorgänge wie bei der deutschen Beteiligung im Kongo oder Libanon in keiner Weise ein drittes Mal mit. Es könne nicht sein, dass ein deutscher Einsatz so lange herbeigeredet werde, bis er sich angeblich nicht mehr vermeiden lasse. Vgl. Widerstand gegen erneuten Afrika-Einsatz, in: Die Welt vom 30. November 2006.

74 Vgl. Berlin schweigt über Soldatenzahl, in: FAZ vom 11. Mai 2006.

75 Vgl. Peter Schmidt, a.a.O., S. 56. 
handelt es sich jedoch um nichts wesentlich Neues. Die militärischen Auslandseinsätze auf dem Balkan sowie das Engagement in Afghanistan wurden von der Bündnisräson im Rahmen der NATO vorangetrieben. Beim Einsatz im Kongo war es die Notwendigkeit, die neue Afrika-Strategie der EU durch Taten zu unterstützen und die Glaubwürdigkeit der EU als außen- und sicherheitspolitischer Akteur unter Beweis zu stellen.

Eine interessante hypothetische Frage lautet, ob ein Einsatz der rein deutschen Eingreiftruppe (so wie ursprünglich vorgeschlagen) im Bundestag zustimmungsfähig gewesen wäre. Der oben beschriebene Verlauf der Verhandlungen im Januar 2006 bietet hier eine neue Perspektive: Die innenpolitischen Vorbehalte gegen eine solche Lösung konnten durch die Regierung bei den internationalen Verhandlungen instrumentalisiert werden. Die öffentlich vorgetragenen Zweifel der Parlamentarier spielten eine Rolle bei der Argumentation der Bundesregierung auf internationaler Ebene, warum ein Einsatz der deutschen Truppe nicht möglich sei und es sich um einen breiter angelegten europäischen Einsatz handeln müsse. Genau dies deutet darauf hin, dass dem Bundestag wohl Handlungsspielraum blieb. Zwar nicht im formellen Teil des Entscheidungsprozesses (das heißt ab April / Mai 2006), sondern vorher während der regelmäßigen Konsultationen in den Ausschüssen. Die breit geteilte Zurückhaltung im Bundestag gegenüber dem Kongo-Einsatz konnte die Regierung im internationalen Verhandlungsprozess ausnutzen. Sie hat es jedoch nicht beziehungsweise nicht genügend getan. ${ }^{76}$

Des Weiteren ist auf die direkte Einbeziehung von Abgeordneten in die Verhandlungen hinzuweisen. So reisten nach verfügbaren Angaben circa zehn Abgeordnete im Frühjahr 2006 in den Kongo. Es handelte sich entweder um länger geplante Reisen oder um Informationsreisen aus aktuellem Anlass. Die Berichte dieser Abgeordneten hatten in den Debatten zumeist ziemliches Gewicht. Sehr wichtig war auch die routinemäßige auswärtige Sitzung des Verteidigungsausschusses in Brüssel. Obwohl von den Abgeordneten unterschiedlich wahrgenommen, konnte das Treffen der Abgeordneten mit Javier Solana und Vertretern des EU-Militärstabs bei der Kritik der angeblich mangelhaften Vorbereitung des Einsatzes im politischen Streit instrumentalisiert werden.

\section{Schlussfolgerung: Anpassungen notwendig?}

Belegen die Erfahrungen aus dem Entscheidungsprozess über den Kongo-Einsatz, dass der konstitutive Parlamentsvorbehalt im Zeitalter multilateraler Verbände zugunsten schneller Entscheidungen verändert werden muss? Diese Frage ist aus folgenden Gründen zu verneinen, wobei das Kongo-Beispiel eher auf die Schwächen der bisherigen Argumentationsmuster verweist.

Erstens handelte es sich um keinen Einsatz einer Eingreiftruppe, sondern um eine ad hoc-Lösung. Es ist daher problematisch, Schlüsse für eine künftig nötige schnelle Entscheidung über den Einsatz eines multinationalen Verbandes ziehen zu wollen. Der Entscheidungsprozess war äußerst langwierig, da die Wahlen im Kongo mehrmals verschoben wurden. Der Bundestag hatte dadurch auch genügend Zeit für Diskussionen, vor allem in den Ausschüssen. Diese wurde auch genutzt, denn nahezu in jeder Sitzung des Verteidigungs-

76 Vgl. ebenda; Denis M. Tull, a.a.O., S. 69. 
ausschusses stand der Sachstand hinsichtlich einer Beteiligung der Bundeswehr im Kongo auf der Tagesordnung.

Zweitens zeigt der Entscheidungsprozess, dass ein „Entsendeausschuss“ keine Lösung (zumindest beim Kongo-Fall) gebracht hätte. Ein solcher Vorschlag77 zielt eher auf die Überwindung der bestehenden Rivalität zwischen dem Auswärtigen und dem Verteidigungsausschuss in der Frage der Auslandseinsätze. Die bestehenden Ausschüsse sind langfristig mit dem multilateralen Rahmen der Auslandseinsätze vertraut, betreiben eine eigene unabhängige Informationsbeschaffungspolitik (so wie der Verteidigungsausschuss am 8. März in Brüssel) und können somit eigene Akzente in der Diskussion setzen. Ein Entsendeausschuss, der lediglich auf die Informationen der Regierung angewiesen wäre, würde die Position des Bundestages faktisch schwächen. Es ist auch völlig unklar, ab wann sich ein solcher Entsendeausschuss mit einem Auslandseinsatz beschäftigen sollte, denn nach dem Regierungsbeschluss ist es wohl zu spät, Einfluss auf die Regierung ausüben zu wollen.

Drittens war die Entscheidung über den Einsatz im Kongo ein Kompromiss zwischen dem Druck von außen und innenpolitisch bedingter Zurückhaltung ${ }^{78}$, die jedoch nur durch den informellen Beratungsprozess ${ }^{79}$ vor der konstitutiven Beschlussfassung kanalisiert und zum Ausdruck gebracht werden konnte. Die Ausschüsse des Bundestages stellten in dieser Hinsicht die Plattform für verschiedene Meinungen dar. Die Fallstudie demonstriert, dass der konstitutive Parlamentsvorbehalt mehr umfasst und umfassen muss als das im PBG geregelte Verfahren einer formellen „Absegnung“ der Regierungsvorlage.

Damit zeigt das Beispiel des Kongo-Einsatzes zwar, dass der konstitutive Parlamentsvorbehalt dem Bundestag eine Mitwirkungsmöglichkeit de facto nur vor dem Regierungsbeschluss sichert, gleichzeitig werden aber auch die Schwächen der Forderung nach noch früherer Einbindung des Parlamentes in die Entscheidungsfindung der Regierung deutlich. Die Gefahr ist groß, dass nicht ausgereifte Vorstellungen innerhalb der Exekutive als wechselhafte Meinungen beziehungsweise Wortbrüche ausgelegt werden. Hierfür steht beispielhaft der politische Lernprozess von Verteidigungsminister Jung, dessen Informationspolitik gegenüber dem Bundestag immer restriktiver wurde. Eine frühere und intensivere Einbindung des Bundestages und der Ausschüsse setzt also eine wesentlich kooperativere Kultur der Zusammenarbeit in den Ausschüssen voraus, in der die Informationen aus dem Meinungsbildungsprozess nicht im öffentlichen parteipolitischen Streit ausgenutzt werden - eine Forderung, die im Lichte der vorher geschilderten Abläufe wohl unrealistisch ist.

Damit Deutschland nächstes Mal nicht wieder gegen seinen Willen in einen Einsatz hineinmanövriert wird, bedürfte es einer deutlicheren Definition und Verfolgung von Interessen und besserer Abstimmung zwischen den einzelnen Akteuren anstatt einer institutionellen Anpassung, etwa in Form eines Entsendeausschusses.

77 Entweder in der Form, wie er von der FDP in ihrem „Entwurf eines Gesetzes zur Mitwirkung des Deutschen Bundestages bei Auslandseinsätzen der Bundeswehr“, BT-Drs. 15/1985, vorgeschlagen wurde, oder wie ihn Noetzel und Schreer fordern, vgl. Timo Noetzel / Benjamin Schreer, Vernetzte Kontrolle, a.a.O., S. $41 \mathrm{f}$.

78 Vgl. Peter Schmidt, a.a.O., S. 57.

79 Vgl. Florian Schröder, a.a.O., S. 134 ff. 